\title{
ENDOMETRIOSIS IS ASSOCIATED WITH HYPOTHYROIDISM IN MARRIED WOMEN
}

\author{
Rose Mary Jacob Vatakencherry1, Saraswathy L2, Radhamany K³, Sajitha Krishnan4, Sundaram K. ${ }^{5}$
}

${ }^{1}$ Clinical Assistant Professor, Department of Physiology, Amrita School of Medicine, Amrita Institute of Medical Sciences, Amrita Vishwa Vidyapeetham University.

${ }^{2}$ Clinical Professor and HOD, Department of Physiology, Amrita School of Medicine, Amrita Institute of Medical Sciences, Amrita Vishwa Vidyapeetham University.

${ }^{3}$ Clinical Professor and HOD, Department of Obstetrics and Gynaecology, Amrita School of Medicine, Amrita Institute of Medical Sciences, Amrita Vishwa Vidyapeetham University.

${ }^{4}$ Clinical Professor, Department of Biochemistry, Amrita School of Medicine, Amrita Institute of Medical Sciences, Amrita Vishwa Vidyapeetham University.

5 Professor and HOD, Department of Biostatistics, Amrita School of Medicine, Amrita Institute of Medical Sciences, Amrita Vishwa Vidyapeetham University.

\section{ABSTRACT}

\section{BACKGROUND}

Endometriosis is a gynaecological condition in which cells in the endometrium appear and flourish outside the cavity of uterus, mostly on the ovaries. Other sites include fallopian tubes and ligaments supporting uterus. Its exact cause is still unknown. Endometriosis shows elevated levels of cytokines, decreased apoptosis and cell mediated abnormalities. Women with endometriosis were found to have higher rates of autoimmune disorders including hypothyroidism.

\section{OBJECTIVE}

To compare the incidence of hypothyroidism in married women with endometriosis (Study group) and married women without endometriosis (Control group).

\section{METHODS}

Sixty married women with endometriosis and sixty married women without endometriosis (Age group: 20-40 years) attending Gynaecology Outpatient Department of Amrita Institute of Medical Sciences, Kochi, from October 2012 to March 2013 were enrolled in the case control study. Serum levels of free T4 and TSH were estimated by chemiluminescent Immunoassay method.

\section{STATISTICAL ANALYSIS}

Student's ' $\mathrm{t}$ ' test was done using IBM SPSS Statistics 20.0; $\mathrm{p}$ value $<0.05$ was considered as statistically significant.

\section{RESULTS}

$78.33 \%(47)$ of the women in the study group were hypothyroid. In the control group, only $13.33 \%$ (8) had hypothyroidism (p value-<0.001).

\section{CONCLUSION}

Incidence of hypothyroidism was higher in married women with endometriosis when compared to control group. Screening for thyroid diseases in endometriosis is indicated.

\section{KEYWORDS}

Endometriosis, Hypothyroidism, Married Women.

HOW TO CITE THIS ARTICLE: Vatakencherry RMJ, Saraswathy L, Radhamany K, et al. Endometriosis is associated with hypothyroidism in married women. J. Evolution Med. Dent. Sci. 2016;5(29):1497-1499, DOI: 10.14260/jemds/2016/352

\section{INTRODUCTION}

Endometriosis is a gynaecological condition characterised by the presence of endometrial tissue in ectopic sites outside uterus.

Financial or Other, Competing Interest: None.

Submission 19-01-2016, Peer Review 16-03-2016,

Acceptance 22-03-2016, Published 11-04-2016.

Corresponding Author:

Dr. Saraswathy $L$

Clinical Professor and HOD

Department of Physiology,

Amrita School of Medicine,

Amrita Institute of Medical Sciences,

Kochi, Kerala, India.

E-mail: saraswathyl@aims.amrita.edu

DOI: $10.14260 /$ jemds/2016/352
The term endometriosis is derived from the ancient Greek words; "End" meaning inside, "Metra" meaning womb and "Osis" meaning disease, problem or abnormality.

Endometriosis occurs in roughly $6-10 \%$ of women.(1) It is primarily a disease of reproductive years.(2) In infertility population incidence is higher, accounting for the single most frequent cause of infertility.(1) Endometriosis is most often found in the ovaries. Other sites include fallopian tubes, ligaments supporting uterus. Its cause is still unknown. One theory is that during menstruation some of the menstrual tissue backs up through the fallopian tubes into the abdomen, where it implants and grows (Implantation/transplantation theory).(3) Another theory suggests that endometriosis may be a genetic process - predisposing certain families to endometriosis. Symptoms include pelvic pain in various 
manifestations.(4) constipation.(5) chronic fatigue.(6) Diagnosis is based on patient's medical history, complete physical examination including pelvic examination. Diagnosis is complete only by laparoscopy. Endometriosis is treated by hormone suppressives.

\section{BACKGROUND}

Endometriosis shows elevated levels of cytokines, decreased apoptosis and cell mediated abnormalities showing its autoimmune aetiology. Women with endometriosis were found to have higher rates of autoimmune disorders including hypothyroidism.(6)

\section{OBJECTIVE}

To compare the serum levels of free T4 and TSH in married women with endometriosis (Study group) and married women without endometriosis (Control group) in the age group 20-40 years.

\section{MATERIALS AND METHODS \\ Study Design}

Study was carried out as a case control study in the Department of Obstetrics and Gynaecology, Amrita Institute of Medical Sciences, Kochi, Kerala, from Sept 1st 2012 to March 31st 2013.

\section{Study Subjects Cases}

Sixty married women with endometriosis were selected for the study based on the inclusion criteria. The diagnosis of endometriosis was based on the symptoms and pelvic ultrasound scan.

\section{Controls}

Sixty married women without endometriosis were selected from age matched female bystanders of patients, female subjects coming for health check-up in comprehensive health check-up clinic in AIMS, nursing staff, paramedical staff and paramedical students of AIMS who had regular menstrual periods, non-pregnant and not lactating. Those who had chronic illness were excluded. Anthropometric measurements including height in meters and weight in kg were measured.

Each subject was interviewed and a standard questionnaire was filled up containing information on demographics, anthropometric profile, past medical history and biochemical parameters. A detailed physical examination including a pelvic examination and a pelvic ultrasound was done to confirm the presence of endometriosis. All participants gave a written informed consent to take part in the study, which was approved by the Institution Ethics Committee.

\section{Inclusion Criteria}

1. Diagnosis of endometriosis based on symptoms and pelvic ultrasound; 2 . Age between 20 and 40 years.

\section{Exclusion Criteria}

1. Age group below 20 and above 40 years; 2. Current pregnancy or nursing; 3 . Conditions known to affect thyroiddiabetes, hypercholesterolemia and autoimmune diseases like psoriasis.

\section{Biochemical Analysis}

Peripheral venous blood samples $(4 \mathrm{~mL})$ were collected randomly from all the participants. Serum was separated by centrifuging at $3000 \mathrm{rpm}$ for 5 minutes. Blood glucose was estimated by hexokinase method on Olympus AU2700 analyzer. HDL, LDL, VLDL and triglycerides were estimated by enzymatic method on Olympus AU2700 analyzer. Thyroid Function Test (TFT) was done by Chemiluminescent Immunoassay (CLIA) on Abbott Architect plus analyzer. Chemiluminescence Kit (CLIA Kit) is a kind of sensitive Kit, which uses Streptavidin-HRP as enzyme and enhanced ECL chemiluminescent system as substrate reagent.

The assay processes of CLIA Kit is listed below: at first, test samples are added into black opaque microplates which were coated with antibodies; secondly, horseradish peroxidase complex conjugates with specific antibody added and then the un-reacted ingredients were washed away; thirdly, Luminol chemiluminescent substrate will be added into the microwells and then Relative Luminosity Values (RLU) will be scanned by photon counter reader. Chemiluminescent detection technology has been considered as the most promising laboratory diagnostic methods because of its high sensitivity and no radioactive contamination.

\section{Statistical Analysis}

Student's ' $t$ ' test was done using IBM SPSS Statistics 20.0. For all the continuous variables, the results were given in Mean \pm SD and for all categorical variables the results were given as percentage. To compare the averages of continuous variables between two groups, those following normal distribution, independent sample ' $t$ ' test was performed. Pearson's chisquare test was applied to test the relationship between two categorical variables. P-value of $<0.05$ were considered as statistically significant.

\section{RESULTS}

A total of 120 married women (60 with endometriosis and 60 without endometriosis) participated in the study. Average age of married women in study and control group were $34.54 \pm 6.34$ and $35.84 \pm 5.51$, respectively.

\begin{tabular}{|c|c|c|c|}
\hline \multirow{2}{*}{ Category } & \multicolumn{2}{|c|}{ Hypothyroidism } & \multirow{2}{*}{$\begin{array}{c}\text { p- } \\
\text { value }\end{array}$} \\
\cline { 2 - 3 } & $\begin{array}{c}\text { Positive } \\
\mathbf{n}(\%)\end{array}$ & $\begin{array}{c}\text { Negative } \\
\mathbf{n}(\%)\end{array}$ & \\
\hline Endometriosis (+) & $\begin{array}{c}47 \\
(78.33)\end{array}$ & $\begin{array}{c}13 \\
(21.67)\end{array}$ & \multirow{2}{*}{$<0.001$} \\
\hline Endometriosis (-) & $8(13.33)$ & $\begin{array}{c}52 \\
(86.67)\end{array}$ & \\
\hline
\end{tabular}

Table 1: Incidence of Hypothyroidism in Two Groups

\section{CONCLUSION}

The incidence of hypothyroidism was more in the study group (78.33\%) than in the control group (13.33\%) (p value-<0.001). Screening for thyroid disturbances in women with endometriosis is indicated.

\section{DISCUSSION}

Our results showed significant increase in hypothyroidism in women with endometriosis. The overall frequency of hypothyroidism was $78.33 \%$ (47) in women with endometriosis and $13.33 \%$ (8) in women in the control group. According to these results, married women with endometriosis are at a higher risk of having hypothyroidism 
and screening for this problem is therefore justified in these women.

The results of the present study are in accordance from those reported by Sinaii et al. (2002), who concluded that women with endometriosis have a higher rate of hypothyroidism compared with the published rates of hypothyroidism in the general US female population $(9.6 \%$ versus $1.5 \%, P$. 0.0001 ).

The strength of the current study is that the diagnosis of hypothyroidism were by serum measurements of thyroid hormone in accordance with strict criteria and the study did not simply rely on information provided by the patients. Furthermore, the control group consisted of women from the same geographical region with similar demographic characteristics.

\section{ACKNOWLEDGEMENTS}

We thank Lord Almighty for the divine guidance in carrying out this study. We sincerely thank Dr. Prem Nair, Medical Director and Dr. Prathapan Nair, Principal, Amrita School of Medicine, Amrita Institute of Medical Sciences, Kochi, for giving us the permission and all the facilities to do the study. We extend our sincere gratitude to Dr. Subhakumari K N, Clinical Professor and HOD-Department of Biochemistry for her help without which we would not have completed the study. We also extend our sincere gratitude to the entire faculty and all the non- teaching staff members of the Department of Physiology, Obstetrics and Gynaecology, Biochemistry and Biostatistics; Amrita Institute of Medical Sciences and Research Centre; Kochi, for their co-operation, support and encouragement throughout the study.

\section{REFERENCES}

1. Bulletti C, Coccia ME, Battistoni S, et al. Endometriosis and infertility. Assist Reprod Genet 2010;27(8):441-7.

2. Nothnick WB. The emerging use of aromatase inhibitors for endometriosis treatment. Reprod Biol Endocrinol 2011;9:87.

3. Fauser BC, Diedrich K, Bouchard P, et al. Contemporary genetic technologies and female reproduction. Hum Reprod Update 2011;17(6):829-47.

4. Endometriosis; NIH September, 2002;02-2413.

5. Ballard $\mathrm{K}$, Lane $\mathrm{H}$, Hudelist $\mathrm{G}$, et al. Can specific pain symptoms help in the diagnosis of endometriosis? a cohort study of women with chronic pelvic pain. Fert Steril 2010;94(1):20-7.

6. Sinaii N, Cleary SD, Ballweg ML, et al. High rates of autoimmune and endocrine disorders, fibromyalgia, chronic fatigue syndrome and atopic diseases among women with endometriosis: a survey analysis. Human reproduction 2002;17(10):2715-24. 